\title{
Effect of the Base Cavity with an Irregular Shape on the Aerodynamic Drag of Projectile
}

\author{
Haibo Lu \\ Nanjing Artillery Academy, Nanjing, 211132, China \\ Ihbboo@sohu.com
}

Keywords: Projectile with base cavity; Irregular shape; Aerodynamic drag reducing; Numerical simulation

\begin{abstract}
This paper focus on the improvement of the aerodynamic drag reducing of the base cavity configuration on the projectile under supersonic flow condition. Projectile with two sorts of irregular shape of the base cavity which is distinguished from the traditional column base cavity is investigated numerically. Flow field parameters and the aerodynamic drag of the projectile were obtained. Results show that, by the changing of the base cavity shape, the aerodynamic drag of the projectile could be weakened.
\end{abstract}

\section{Introduction}

Base cavity configuration which is used on projectile in order to increase the range of the gun is mentioned by researchers of the United States firstly in the last century sixtieths. Investigations in the field continued up to now [1-8]. Nowadays, projectile with base cavity is used wildly on kinds of medium and large caliber artillery in countries around the world.

In this paper, two sorts of irregular shape of the base cavity which is distinguished from the traditional column base cavity of the projectile are investigated numerically. The influence of the base cavity shape on the aerodynamic drag of projectile is discussed.

\section{Configuration of the Base Cavity with Abnormity Shape}

The traditional base cavity shape is the column one. Two sorts of irregular shape of the base cavity are shown in Fig. 1. The base cavity is half column and half truncated-cone with positive (shape "S") or negative (shape "F") inclination.

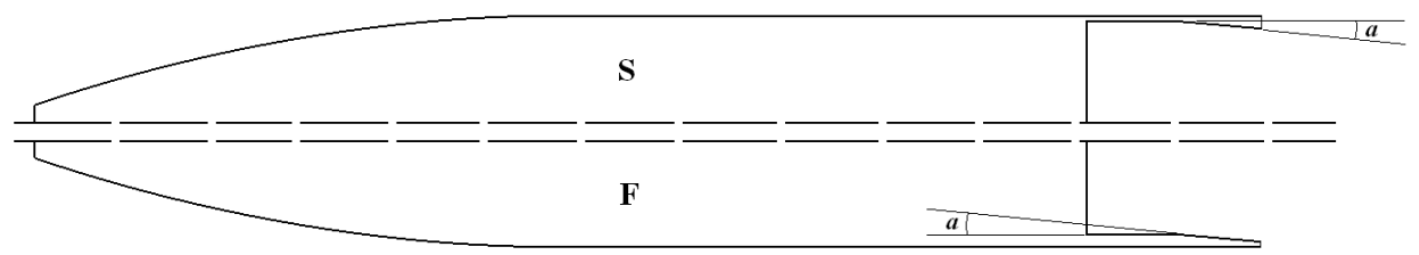

Figure 1. Configuration of the projectile with the base cavity

\section{Computation Scheme}

Governing Equation The axisymmetric N-S equation is given by [9]

$$
\partial U / \partial T^{+} \partial F(U) / \partial x+{ }^{\partial G(U)} / \partial r+1 / r \cdot \partial H(U) / \partial r=1 / \operatorname{Re} \cdot\left(\partial F_{v}(U) / \partial x+{ }^{\partial G_{v}(U) / \partial r}+1 / r \cdot \partial H_{v}(U) / \partial r\right)
$$

where $U$ is the conservation variable, $F, G$ and $H$ are the inviscid terms, $F_{v}, G_{v}$ and $H_{v}$ are the viscous terms. The $k-\varepsilon$ turbulence model [10] is used in the simulation. The convective terms are 
approximated using the Van Leer [11] splitting method and the central difference method is used for the viscous terms. The LU-SSOR scheme is used for the time integration.

Boundary Conditions and Grid. The flow conditions are given in Table 1. The wall boundary condition is assumed to no-slip and adiabatic.

Table 1 Boundary conditions

\begin{tabular}{|c|c|c|}
\hline Free stream parameter & Unit & Value \\
\hline Mach Number $(M a)$ & ---- & 1.97 \\
\hline Pressure $\left(p_{\infty}\right)$ & $\mathrm{Pa}$ & 101325 \\
\hline Temperature $\left(T_{\infty}\right)$ & $\mathrm{K}$ & 300 \\
\hline
\end{tabular}

As shown in Fig. 2, the body-fitted grid of the projectile with base cavity (shape "F") is given.

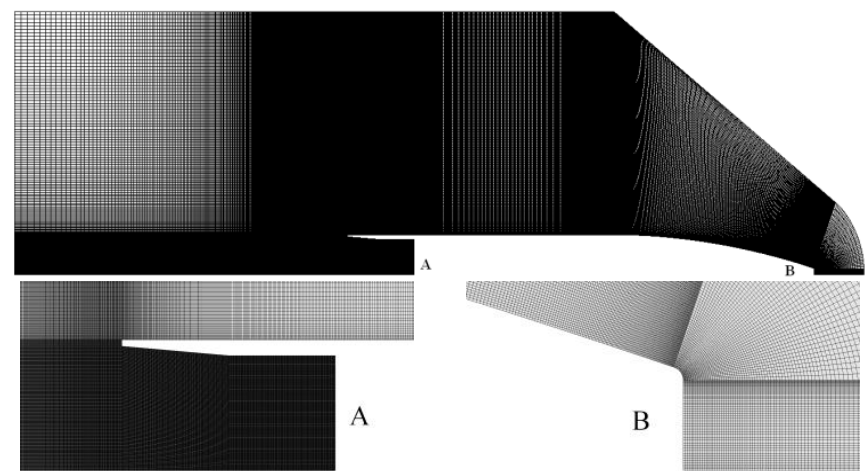

Figure 2. Schematic of the simulation model grid (Shape "F")

\section{Results and Discussion}

Flow Field. The distribution of the Ma and streamline of projectile with each shape base cavity is given in Fig. 3. As shown, distributions of Ma and streamline of all projectiles are similar. With the same warhead shape, the front part of the flow field of each shape is the same too. Main difference of each flow field locates at the bottom of the projectile. With different base cavity shape, there is a little difference between circumfluence regions inside the base cavity.

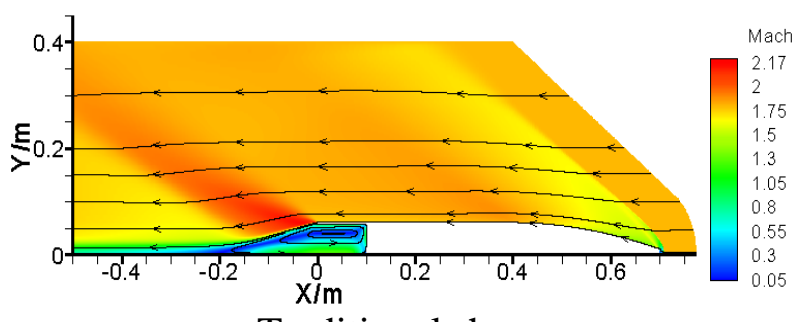

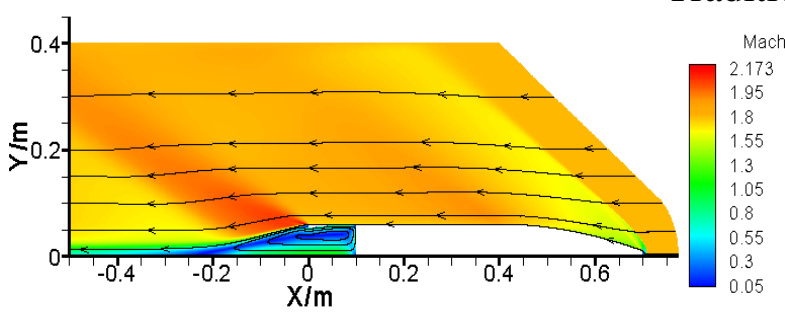

Shape "S"

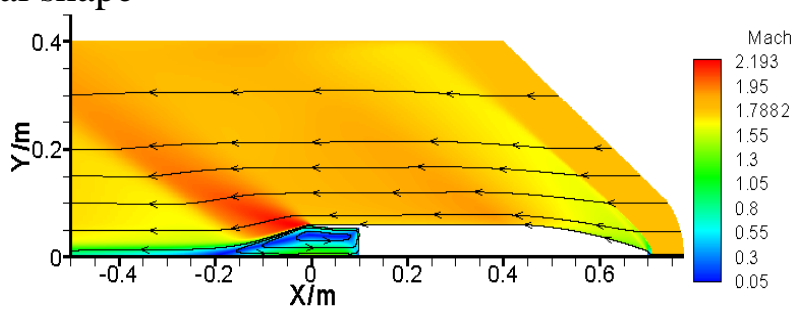

Shape "F"

Figure 3. Distribution of the $M a$ (streamline) 
As shown in Fig. 4 and Fig. 5, the distribution of the temperature and the pressure of projectile with shape "S" and " $F$ " is given. Distributions of temperature and pressure of all projectiles are similar.

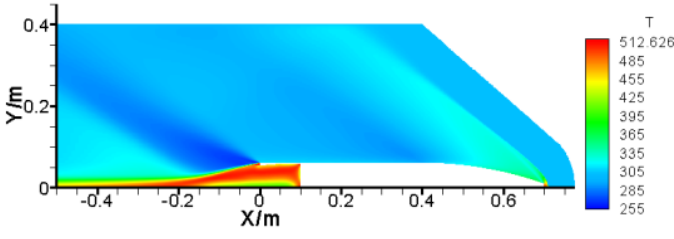

Shape "S"

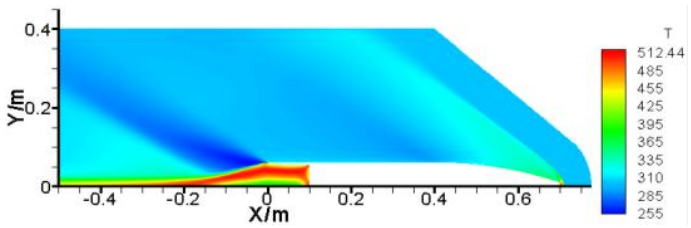

Shape "F"

Figure 4. Distribution of temperature

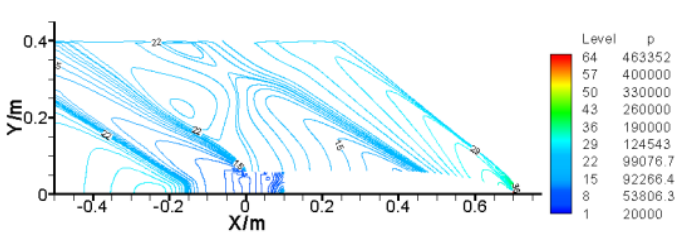

Shape "S"

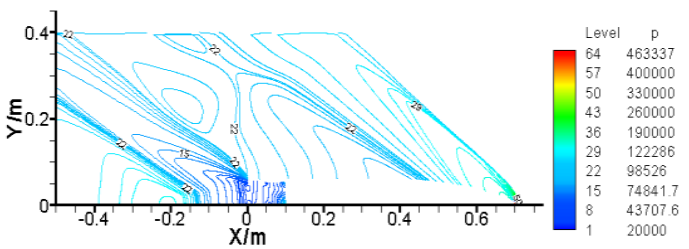

Shape "F"

Figure 5. Distribution of pressure

Aerodynamic Drag. Though the flow field of the projectile with base cavity of the three shapes is similar, the aerodynamic drag coefficient is different. As shown in Table 2, projectile with shape "S" base cavity has the minimal aerodynamic drag coefficient. It is obviously that base cavity with shape "S" has the best aerodynamic drag reducing efficiency. Projectile with shape "F", it's coefficient of drag even larger than the traditional cavity shape, this means that shape " $F$ " is not an optimized configuration for the projectile to reduce the aerodynamic drag.

Table 2 Aerodynamic drag coefficient of each projectile

\begin{tabular}{|c|c|c|c|}
\hline Cavity shape & $\mathrm{S}$ & Cylinder & $\mathrm{F}$ \\
\hline$C_{d}$ & 0.3071 & 0.3267 & 0.3326 \\
\hline
\end{tabular}

The distribution of pressure along the base wall of projectile with each shape base cavity is shown in Fig. 6 . At the centre of the base wall of the cavity $(\mathrm{Y}=0 \sim 0.011 \mathrm{~m})$, the shape " $F$ " has largest pressure along the wall which the shape " $\mathrm{S}$ " is smallest. But in this region, area of it is small. In the rest of the vast area ( $\mathrm{Y}=0.011 \mathrm{~m} \sim 0.058 \mathrm{~m})$, pressure along the shape " $\mathrm{S}$ " is larger than the traditional one. And pressure along the shape " $\mathrm{S}$ " is larger than the shape " $\mathrm{F}$ ", too, until $\mathrm{Y}=0.044 \mathrm{~m}$. Though at the centre and the edge of the base wall, the pressure along the wall of shape " $F$ " is higher than shape " $\mathrm{S}$ ", the total effect is that the back pressure of shape " $\mathrm{S}$ " is higher than shape "F". And comparing the total area of the cavity base wall, shape "S" is larger than shape "F"; this is benefit to get higher back pressure.

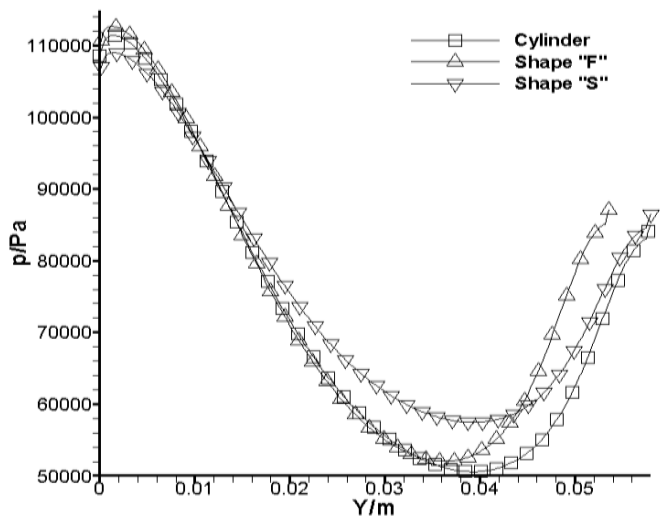

Figure 6. Distribution of pressure along the base wall 


\section{Conclusions}

The effect of base cavity on aerodynamic drag of the projectile can be optimized by changing the configuration of the base cavity.

The changing of the base cavity configuration causes to little alternation on the flow field inside the cavity, but lead to a notable efficiency on its aerodynamic drag.

The base cavity with the shape half column and half truncated-cone with positive inclination (shape "S") make the best performance on aerodynamic drag reducing of the projectile.

On the contrary, the base cavity with half column and half truncated-cone with negative inclination (shape " $F$ "), its efficiency on aerodynamic drag reducing is even worse than the traditional columned base cavity.

\section{References}

[1] J. Howell, Sims-Williams D, Sprot A, et al. Bluff body drag reduction with ventilated base cavities, SAE Technical Papers, 5(2012) 152-160.

[2] J.Y. Cao, C.J. Lu, Y. Chen, X. Chen and J. Li, Research on the base cavity of a sub-launched projectile, Journal of Hydrodynamics, 24 (2012) 244-249.

[3] A. Ibrahim, A. Filippone, Supersonic aerodynamics of a projectile with slot cavities, Aeronautical Journal, 114 (2010) 15-24.

[4] Z.Y. Wang, The effect about projectile of base cavity with side holes to flight drag under supersonic flow, ACTA aerodynamica sinica, 15(1997) 502-506. (In Chinese)

[5] M. G. Edgar, Preliminary investigation of effectiveness of base bleed in reducing drag of blunt-base bodies in supersonic stream, NASA RM26, 1971.

[6] R. D. Elizabeth, The effectiveness of base-bleed in reducing drag of boat-tailed bodies at supersonic velocities, PB157711, 1970.

[7] H.Z. Wei, H.S. Zhu, The design theory of projectile. Beijing: National Defense Industry Press, 1985. (In Chinese)

[8] H.B. Lu, X.R. Shi, S.Y. Tian and Q. Gan, Research on the Effect of the Base Cavity with Different Length Upon the Projectile, Advances in Computer Science Research, 25(2015) 22-25.

[9] X.S. Wu, The numerical investigation on flow field of projectile (with base bleed). Journal of ballistics, 4 (1992) 39-43. (In Chinese)

[10] W.Q. Tao, Numerical Heat Transfer, 2nd ed. Xi' an: Xi'an Jiaotong University Press, 2001 (In Chinese)

[11] X.D. Wang, J.J. Tan, X.H. Lin and Z.H. Tang, Research on parallel numerical simulation of N-S equations based on Van Leer+AUSM scheme, Journal of Astronautics, 31(2010) 986-992. (In Chinese) 